\title{
Value Driven Conceptual Design of Unmanned Air System for a Defence Application
}

\author{
Evangelos Papageorgiou ${ }^{1}$, Murat Hakki Eres ${ }^{2}$ and James Scanlan ${ }^{3}$ \\ Computational Engineering and Design Research Group, Faculty of Engineering and the Environment, University \\ of Southampton, Southampton, SO16 7QF, UK
}

The work presented culminates in the development of a value driven conceptual design assessment framework for a small Unmanned Air System (UAS) to be utilized in a defence application. In the field of Multi-Disciplinary Design Optimisation, most recent systematic search has been devoted to fixed topology parametric geometries, pertaining to a single concept, with very little stress put on the optimization of variable topologies describing alternative design concepts. The search is conducted in a highly novel manner, generating a broad range of combinations of UAS configurations and geometries by systematically searching alternative concepts and design configurations through the parameterization of the aircraft geometric topologies. Moreover, the "value" of proposed solutions is assessed in an objective way both from performance and economic perspectives, while the optimal solution is identified based on the user's needs after relaxing all of the design constraints. During the multi-criteria decision analysis, the quantification/conversion of the linguistic preferences of the user between the various attributes to numerical values has disclosed some deficiencies introduced by the unjustifiable numerical scales used in the Analytic Hierarchy Process (AHP) and a novel value model for consistent value assessment is introduced, synthesizing the AHP assessment methodologies with multi-attribute value-focused analysis.

\footnotetext{
${ }^{1}$ Ph.D. student, Faculty of Engineering and the Environment, Southampton Boldrewood Innovation Campus, Building 176, University of Southampton, Burgess Road, Southampton, SO16 7QF, UK, Tel.:0044 7581812956, email: ep1v12@soton.ac.uk (corresponding author).

2 Senior Research Fellow, Faculty of Engineering and the Environment, Southampton Boldrewood Innovation Campus, Building 176, Room 5025, University of Southampton, Burgess Road, Southampton, SO16 7QF, UK.

3 Professor, Faculty of Engineering and the Environment, Southampton Boldrewood Innovation Campus, Building 176, Room 5027, University of Southampton, Burgess Road, Southampton, SO16 7QF, UK.
} 
Keywords: aerospace conceptual design, value-driven design, unmanned air system, multi-criteria decision analysis, Analytic Hierarchy Process, Multi-attribute Utility Theory. 


\section{Introduction}

According to Wiese [46], the successful exploration of the design space in generic engineering design needs to be a) 'systematic', in the way all potential solutions are proposed and evaluated against the main objectives; b) 'iterative', using both simulation and prototyping to assess them; and c) 'multidisciplinary', encompassing all important considerations across different disciplines. All key system aspects of all lifecycle stages of the designed system, from development and production to its disposal need to be addressed when studying the system, its components and their interactions with the wider environment. As proposed by Hazelrigg [23], through the iterative engineering design process of Fig. 1, several potential design solutions, with different characteristics are generated and evaluated against the primary objectives to select the best one.

In order to locate the best solution, the optimization process should explore the widest possible design space, after removing any design constraints. Moreover, the value driven conceptual design assessment framework should assess the goodness of proposed systems solutions through the use of multiple criteria decision analysis approach addressing all economic and non-economic needs of the user to identify the value-enhancing design(s).

The research presented in this paper addresses a wide range of essential concepts that are needed for successful UAS design. These can be summarized as:

- Employment of a wide range of UAS platforms.

- $\quad$ Quick and efficient conceptual design exploration.

- Use of a system definition that is easily amended and replaceable for higher accuracy.

- Definition of a unit acquisition costing system and operational scenarios, in order to obtain accurate life cycle cost estimates.

- Derivation of a value/utility assessment framework that considers the needs and requirements of customers/stakeholders and all relevant design attributes.

\section{Literature Review}

\section{A. Value Driven Engineering Design}

Engineering design has been studied thoroughly both in academia and industry. Hubka and Eder [24], Pugh [32], Pahl and Beitz [30], Otto and Wood [29], Eggert [18], Ulrich and Eppinger [44] and Ullman [43] introduced several 
systematic applications aiming to accelerate the iterative design process. Cross [12] describes the plan of action for product design, while the evolution of the generic synthesis of the multidisciplinary design problem is described by Antonnson and Cagan [4].

The method of Multidisciplinary Design Optimization (MDO) for engineering design across different functional areas was first applied systematically in the 1960's. Sobieski of NASA Langley Research Centre defines MDO as a 'methodology for design of complex engineering systems that are governed by mutually interacting physical phenomena and made up of distinct interacting subsystems', explaining that 'in their design, everything influences everything' [33]. Several algorithms and MDO methods are applied in engineering design, while the selection of the most appropriate method depends on the specific application.

The systems engineering approach was introduced in the engineering design process by Hazelrigg [22] and is still the dominant integrating framework for engineering design. According to the International Council on Systems Engineering (INCOSE) Systems Engineering Handbook [3], Systems Engineering is a 'discipline that concentrates on the design and application of the whole (system) as distinct from the parts, through an iterative process of topdown synthesis, development and operation of a system that satisfies in a near optimal manner the full range of requirements for it'.

Due to the delays and cost overruns experienced in engineering design as systems became more elaborate and complex, Collopy and Hollingsworth [11] proposed a value driven optimization process. This process followed the systems engineering approach of breaking the system to subsystems and components but flowing down to the subsystems and components instead of design requirements the system objective function. For each subsystem and component, the component attributes are converted through an appropriate composition function to system extensive attributes, which are then input to the value model/objective function to compute a value score for this specific component design. Consequently, Value Driven Design (VDD) is the framework that relaxes all requirements set by systems engineering and focuses on the pursuit of value, identifying the design yielding the highest overall value score. Additionally, all components are monitored in terms of their achieved value score, keeping the whole system in balance. The application of VDD framework has been demonstrated by Castagne et al. [6] for designing aircraft fuselage panels and by Cheung et al. [9] for the design of a propulsion system.

\section{B. Multi-Criteria Decision Analysis}


The identification and structuring of the stakeholder's needs/requirements, covering the complete lifecycle of the designed product, is the first step of the engineering design process. As Keeney [25] points out, these needs will pronounce the values of the user and steer both the collection of information and decision making. Keeney and Raiffa [26] describe the process of constructing a non-unique hierarchy of objectives, with one or more comprehensive and measurable attributes associated to each of them; constituting a complete, operational, decomposable, non-redundant, and minimal full set of attributes as the input of the objective/value function, modelling the overall goodness of a particular design alternative.

Several approaches can be used during the multi-criteria decision making process of evaluating a set of alternatives, that are depicted by a number of attributes with the corresponding objectives being pursued. The maximization of value is fundamental to any decision making problem solving; hence, economic theory reflected in cost contributes pari passu with system performance in the creation of the value model. The most significant approaches in MCDA include ELECTRE (Roy [36]), multi-attribute utility theory (MAUT) (Fishburn [21], Keeney and Raiffa [26]), simple multi-attribute rating technique (SMART) (Edwards [16], Edwards and Barron [17]), Analytic Hierarchy Process (AHP) (Saaty [37]), technique for order preference by similarity to ideal solution (TOPSIS) (Yoon and Hwang [47]) and simple additive weighting (SAW) (Kirkwood and Corner [27]). Collopy [10], surveying some of these MCDA techniques used in the development of the value model for engineering design, concluded that it is the user's point of view, adopted in value modelling, that defines the selection of the most appropriate tools. Consequently, the following MCDA techniques are considered to be the most appropriate for value modelling the UAS user's point of view.

\section{Multi-Attribute Value and Utility Modelling}

Value functions represent the worth the stakeholder gives to achieve a certain value of an attribute and for the multi-attribute case. Usually the additive (preferential) independence among the attributes is assumed, i.e. the preferences of the stakeholder over any subset of attributes are independent of its compliment, yielding the following multi-attribute additive value model:

$$
V\left(X_{1}, X_{2}, \ldots, X_{n}\right)=\sum_{i=1}^{n} k_{i} \cdot V\left(X_{i}\right)
$$

The scaling constants $k_{i}$ are assessed using two approaches, the trade-off and the direct rating approaches, described by Keeney and Raiffa [26] and Dyer and Sarin [15] respectively. The individual value functions $V\left(X_{i}\right)$ of 
the attributes are computed independently with a direct value estimation technique, using three distinctive approaches, the direct rating, direct midpoint and direct ordered metric [21].

Utility and multi-attribute utility theory [21] [26] measure preferences of consequences with one or more dimensions, capturing the stakeholder's attitude towards uncertainty. The preferential independence is also assumed, moreover utility independence, concerning the intensity of preferences of any attribute being independent of the levels of the other attributes, is also assumed. In this case the model is a multiplicative one [26]:

$$
K \cdot U(X)+1=\prod_{i=1}^{n}\left[K \cdot K_{i} \cdot U_{i}\left(X_{i}\right)+1\right]
$$

In this equation, $U$ and $U_{i}$ are the total and individual utility functions respectively, while $K$ and $K_{i}$ are the scaling constants. Multi-attribute utility theory has been used as a standard technique in several applications in research and real-world problems, from the airline industry [7] and earthquake projects [5] to deep-space observation mission [35]. Analytic Hierarchy Process

The analytic hierarchy process (AHP) is based on judgments from a group of experts or decision makers and it applies hierarchical decomposition of a high level objective to lower level sub-objectives as described by Saaty [37]. AHP uses pairwise comparisons between all possible pairs to establish an objective weighting for evaluation of a number of alternatives with respect to several criteria/attributes [38]. Additionally, AHP compensates for any bias or inconsistency through the redundant pairwise comparisons' construction of the judgment matrix, assessing the consistency/validity of the model.

However, AHP lacks the sound theoretical axiomatic foundation of utility theory [45], as described by Dyer [14], while the phenomenon of Rank Reversal could happen, when the ranking between the dominating alternatives changes when other dominated/inferior alternatives are introduced [31]. Another disadvantage of the AHP is the construction of its matrix, which is based on the ambiguous question: 'How much better/more important is attribute/alternative $A_{i}$ than $A_{j}$ ?', assessing the ratio scaled strength of preference. It is however used for defining the problem, considering a large number of attributes, communicating value, identifying differences and similarities between various stakeholders' points of view and aggregating them as presented in [28], [39], and [8]. Nevertheless, it is the synthesis of MAUT with AHP that offers significant benefits in the preferences' assessment, as suggested by Dyer [14], and corroborated by several applications [40], [13], [42]. 


\section{UAS Design Framework}

In the proposed value driven modelling framework, the user of the UAS was assumed as the only stakeholder, while an array of parameters, reflecting performance, economic and other concerns, all measured in incommensurable units, models the relative goodness of any future design. The hierarchy of the objectives/attributes is presented in Fig. 2.

For the purpose of the Value Driven Conceptual Design framework application in the conceptual design of a UAS, the specific UAS category selected is that of a Horizontal Take off/Landing (HTOL) Close-Range Mini-UAV with a total weight under $5 \mathrm{~kg}$, backpacked, carried, easily assembled/disassembled and deployed by no more than two persons, fitted with a camera for surveillance and reconnaissance, electrically driven, and with no undercarriage, similar to the Desert Hawk which was designed by Lockheed Martin and is in extensive military use.

The UAS conceptual VDD framework is presented in Fig. 3. The chosen design variables are adjusted to generate feasible design points in the Define phase of the cycle. Extensive system attributes are calculated in the Analyse phase, such as operational surveillance time, maximum range, maximum endurance, lifecycle cost, and so on. These are used as inputs to the value model during the Evaluate phase. The process carries on, in the Search phase through an optimization algorithm or generation of more design points.

\section{A. Aircraft Geometric Topologies}

In this novel approach, the aircraft geometries are systematically generated by parameterizing geometric topologies as proposed by Sobester [41], allowing for the widest possible exploration of the design space as advocated by the VDD philosophy. The generation and hierarchical coding of topological designs of aircraft are based on fundamental design selections:

(1) Starting from the fundamental requirement of having a wing to provide lift.

(2) Follows the existence (or not) of a fuselage, resulting to an aircraft with fuselage or a flying wing.

(3) Next, the type of fuselage is defined, i.e. a conventional ('monolithic') fuselage or a twin boom fuselage aircraft is obtained.

(4) The existence (or not) of a horizontal stabilizer, distinguishing the type of stabilizer, is the next fundamental selection.

(5) The longitudinal position of the stabilizer (forward or aft). 
(6) Its vertical position, relative to the fuselage (conventional horizontal tail or T-shape tail).

(7) The existence and number of vertical fins.

(8) The position of the propeller (forward or aft).

(9) And finally the selection (or not) of all moving control surfaces generate more aircraft geometries. These selections are presented in Fig. 4.

Hence, a multitude of basic aircraft geometries is generated, described by a hierarchical coding composed as a series of 0's and 1's. This coding is input into the appropriate design models defining the aircraft and estimating its relevant attributes. For example, an aircraft with monocoque fuselage, horizontal tail, one vertical fin, a pusher propeller and no all moving control surfaces would be coded as 111110110, a flying wing with a pusher propeller as 100000010 and an aircraft with a twin boom fuselage, inverted V-shape tail, pull propeller as 110011000 . This representation of a large number of aircraft geometric topologies allows for the shape definition to be input in the design models, which are then scaled through the use of appropriate design variables, such as wing span, wing aspect ratio, horizontal aspect ratio, and so on. Thus, the designer considers numerous advantages and disadvantages of each design choice and explores several UAS configurations, as Raymer [34] describes, identifying a different optimal design depending on his/her preferences and priorities.

\section{B. UAS Design Generation and Analysis}

The UAS design generation and analysis is done in four steps:

(1) Aircraft sizing, including structural analysis, drag calculations followed by engine performance and propulsion, weight and balance, and aerodynamic analysis.

(2) The acquisition cost analysis, using the design parameters, product definition and geometry, along with the material and labour cost rates to calculate the UAS unit and total program cost.

(3) The operational simulation analysis model, calculating the maintenance lifecycle cost and losses due to unreliability failures.

(4) The simulation survivability analysis model, providing estimates of the combat damage cost and associated UAS battle losses. 
A basic validation of the aircraft sizing models was performed based on the Southampton University Laser Sintered Aircraft (SULSA), an unmanned air vehicle whose entire structure was 3D printed as a demonstrator of the laser sintering process flexibility for rapid prototyping. The specific UAV was chosen to check the validity of the sizing models, as its configuration is among the chosen ones, i.e. a monolithic/conventional fuselage, aft V-tail and push propeller configuration; and since its design parameters are within the range of those chosen for this research. A close agreement was found between most of the calculated design parameters and those of SULSA, validating the aircraft sizing models used throughout this research.

In lifecycle cost modelling, it was assumed that the failures due to lack of reliability and scheduled maintenance, along with the survivability related combat damages are the governing factors defining lifecycle cost and operational missions' availability of the UAS fleet. Failures occur due to unreliability of critical subsystems/components modelled by Weibull distributions and through Monte Carlo simulations lifecycle cost and survivability related damage cost and uncertainties are estimated.

The chosen design variables for the design alternatives generation are the wing aspect ratio, wing taper ratio, wing span, wing position, horizontal tail aspect ratio, fin aspect ratio, canard aspect ratio, for the canard configuration, battery capacity (electric propulsion is assumed), and component reliability used for the lifecycle cost calculations, as the probability for any component to remain operative until its scheduled replacement.

\section{Multi-Attribute Norm Value Model}

The array of attributes of Fig. 2, all measured in incommensurable units, cannot be collated into an objective function, hence a value model is employed to assess the user's preferences. The multi-attribute additive value model of equation (1) was developed, with scaling constants $k_{i}$ representing the 'weighting importance' of each attribute within the set/subset it belongs and $V_{i}$ the corresponding value functions of the associated attributes $X_{i}$.

Norm is defined in Merriam-Webster Online Dictionary [2], as 'an average level of development or achievement, something that is usual or expected, an authoritative standard, usually derived from the average or median achievement of a large group'. The assignment of norms of average levels of expectations with respect to the attributes by the user is the basis of this novel multi-attribute norm value model, used both for the scaling constants $k_{i}$ and value functions $V_{i}$ assessments. Thus, as Keeney [25] advocates, the alternative-focused process of selecting the best from what is readily available, is converted to a value-focused process of identifying needs, attributes and values of these attributes that give the user a 'neutral' response, a 50\% satisfaction level, as described by Eres et al. [20] in the Concept Design 
Analysis (CODA) methodology. The major advantage of this value model is that it is an efficient and operational way to evaluate each design point, during the conceptual phase when only basic needs and vague requirements are known and the design is not finalized, with the minimum interaction with the user. Moreover, the objectivity of the evaluation is maintained by capturing the user's preferences, with criteria independent of information, other data available or the proposed alternative solutions.

\section{Value Functions}

Before the conceptual design space exploration starts and subject to the technology readiness level assumed, norm values for all attributes are provided a priori by the user. Next, the relationship type for each attribute is defined, i.e. maximizing, if more is better (for performance attributes), minimizing, if less is better (for cost related attributes) or optimizing, for some specific attribute value. The preferences of the user are qualitatively assessed in terms of the marginal evaluation with respect to each attribute in the language of classical economics, i.e. how much the stakeholder is willing to sacrifice in terms of other attributes for a positive change of this attribute as its value changes, reflected in the slope of the value functions; adjusting them in shape as concave, convex or sigmoidal. A convex value function reflects the preferences of a user who is willing to sacrifice more and more in terms of other attributes for the same positive increment as this attribute's values increase. A concave value function is selected if the user is willing to sacrifice less and less in terms of other attributes for the same positive increment as this attribute's values increase. A sigmoidal shape is selected for mixed preferences, i.e. if the user is willing to sacrifice more and more in terms of other attributes for the same positive increment of this attribute up to the inflection point and less and less beyond that point.

Depending on the previous selections, an appropriate, ready to use, value function is generated from all available, presented in Table 1. In the equations of this Table, $X$ is the input value for any design attribute, $n$ is the norm value of the design attribute assigned by the user (representing the $50 \%$ satisfaction level, for example an endurance when flying at the design speed of $1 \mathrm{hr}) . V_{i n}$ and $V_{f}$ are the initial and final values of the value functions (top and bottom, depending on their type). In the figures of this Table, value functions are plotted against the corresponding design attribute. 


\section{Weighting Factors Assessment}

For the assessment of the weighting importance of each attribute, the AHP method was initially employed to perform pairwise comparisons between the attributes and to assess the consistency of the answers provided by the stakeholder. However, the construction of the AHP matrix is based on the ambiguous question: 'How much better/more important is attribute/alternative $A_{i}$ than $A_{j}$ ?', which assesses the ratio scaled strength of preference. It has been found that this unjustifiable selection of numerical scale, such as those presented by Elliot [19], converting the linguistic response to the above question to ratio scaled numerical values, greatly affects the identification of optimal design. These AHP numerical scales convert the stimuli/psychological perception of strength of preference increments among the various attributes to a response/numerical value through the use of an arbitrary relation. Namely, the integer scale assumes a logarithmic relation between stimuli and response, the balanced scale assumes an even distribution of attribute weights whilst the power scale assumes a geometric relation. In Table 2, for the typical 1-9 numerical scale and a five degree of preference scheme, the corresponding values of the integer numerical scale and power numerical scale are presented (for the power scale obtained as $0-4^{\text {th }}$ powers of $\alpha=\sqrt[5-1]{9}$ i.e. $\left.\sqrt[4]{9}, \sqrt[4]{9}^{1}, \sqrt[4]{9}^{2}, \sqrt[4]{9}^{3}, \sqrt[4]{9}^{4},[19]\right)$. Hence, it is noticed that they quantify exactly the same linguistic responses to different numerical values. For instance the verbal response: ' $A_{i}$ is much more important to attribute $A_{j}$ ' is converted to a numerical value of 7 with integer scale and to a value of 5.2 with power scale, with the integer scale favouring more the most important to the user attributes and weighing less the least important ones.

Due to the absence of justified criteria for selecting a particular scale, converting the linguistic answer/stimuli to a numerical value/response, Elliot [19] suggests to study the distribution of weights and level of consistency, obtained by different scales used in AHP, to select the best one. In order to study the distribution of weights for the integer, balanced and power scales, all possible combinations of weighting scales were obtained and presented (in this order) in Fig. 5, for a nine degree preference scheme between three attributes, similar to Fig. 1 presented by Elliot [19]. In this figure, the coordinates of each point are the weights of the two attributes, while the third can be computed since their sum is always equal to unity. Thus, these three scales can be compared in terms of the number of distributed points and existence of sparse regions, in order to select the scale with the highest number of points and the least sparse regions.

Elliot identifies as the most preferable scale, based on the distribution of points, the power scale; however in Fig. 5 the following are noticed: 
- The integer scale gives a high number of points in the most extreme values of weights as expected, considering the distribution of values of the integer scale: $1,2,3,4,5,6,7,8,9$, that weighs more the most important to the user attributes and less the least important ones, as compared to the distribution of values of the balanced scale: $1,11 / 9,4 / 3,13 / 7,7 / 3,3,4,17 / 3,9$ and the distribution of values of the power scale: 1 , 1.316, 1.732, 2.28, 3, 3.948, 5.196, 6.84, 9. Therefore, the balanced and power scales are more evenly distributed than the integer scale.

- Comparing the sparse regions and clustering obtained with the three scales; the power scale fails to cover a larger area in the graph than the integer and balanced scales which produce a definitely higher number of points and less clustering. This observation is not in agreement with Elliot [19] who notices larger sparse regions and clustering of the weights in integer and balanced scales.

Furthermore, to explore the use of different scales in AHP and based on the value model using the two scales, integer and power scale defined in Table 2, a multi-disciplinary optimization was performed. It was found that exactly the same verbal responses/preferences provided by the user, when converted to numerical weights through different AHP scales produced different optimal aircraft designs. Hence, through the use of integer scale, a V-shape tail, push propeller, conventional fuselage with a wing span $1.5 \mathrm{~m}$ aircraft was identified as optimal, while through the power scale, an aircraft of T-shape tail, pull propeller, conventional fuselage with a wing span of 1.25m.

The problem of converting verbal preference responses between attributes to numerical values using some unjustifiable scale in AHP for the calculation of weighting factors can be tackled if instead of performing pairwise comparisons between abstract attributes, the user is forced to compare specific value differences of these attributes. To assess the strength of preferences (value differences) between alternatives, based on the theory of measurable multi-attribute value functions presented by Dyer [15], apart from preference independence, the assumption of weak difference independence is made, i.e. that the order of preference differences between pairs of each attribute is independent of the levels of the other attributes.

The attribute norms, already used for the value functions assessment, are again employed in a direct rating approach to compute the scaling constants by assessing the relative importance of: 
- The preference difference between a design with attribute $x_{i}$ at the norm, $x_{i}^{1 / 2}$, and all other attributes $\bar{x}_{i}$ at their worst value, $\bar{x}_{i}^{o}$, and the design with attribute $x_{i}$ at the worst or best (value of 0 or 1 , depending on the form of value function), $x_{i}^{*, o}$, and all other attributes $\bar{x}_{i}$ at their worst value, $\bar{x}_{i}^{o}$.

- And the preference difference between a design with attribute $x_{j}$ at the norm, $x_{j}^{1 / 2}$, and all other attributes $\bar{x}_{j}$ at their worst value, $\bar{x}_{j}^{o}$, and the design with attribute $x_{j}$ at the worst or best, $x_{j}^{*, o}$, and all other attributes $\bar{x}_{j}$ at their worst value, $\bar{x}_{j}^{o}$.

Through this relative importance assessment of changes to the norm value of any two attributes, the use of any numerical scale can be avoided. Each comparison is represented by equation (3), used to assess the ratio of the weighting factors:

$$
\frac{k_{i} \cdot\left(V_{i}\left(x_{i}^{0.5}\right)-V_{i}\left(x_{i}^{0, *}\right)\right)}{k_{j} \cdot\left(V_{j}\left(x_{j}^{0.5}\right)-V_{j}\left(x_{j}^{0, *}\right)\right)}=\frac{k_{i}}{k_{j}}=c_{i j}
$$

In Table 3, instead of comparing abstract attributes, each cell is the ratio of relative importance/preference of a change to the norm value of row attribute to the change to the norm value of column attribute (from their lowest/highest values). Following the methodology of AHP, several pairwise comparisons are performed, not only to compute the values of the weighting factors, but also assess the consistency of the answers provided by the stakeholder.

As presented in the last two sections, this novel multi-attribute norm value model, based on the qualitative assessment of the user's preferences and the quantitative assignment of neutral values of attributes as norms, allows for the objective evaluation of all design alternatives during the conceptual phase, independent of information, with the minimum interaction with the user. Moreover, the deficiency introduced by the unjustifiable selection of numerical scale used in AHP is solved through the synthesis of AHP with the multi-attribute value functions.

\section{Multi-Attribute Utility Model}

Multi-attribute utility theory is another appropriate tool for dealing with problems when more than one attributes are required to address the multiple objectives. For the UAV design framework, the process followed and all questions posed are as described by Keeney and Raiffa [26]. Preferential independence and utility independence were assumed, reducing the model to the multiplicative model of equation (2), with $K$ and $K_{i}$ scaling factors and $U_{i}$ the utility function for each attribute $X_{i}$. 
Utility functions for all attributes were assessed through a series of certainty to lottery equivalents, after standard parametric families of utility functions were selected. The scaling factors of the multiplicative model were evaluated with independent equations, generated from certainty and probabilistic considerations. The utility model requires extensive interaction with the user to assess their risk attitude, which is not always possible, especially during the conceptual design phase. The decision maker has to be open-minded, interested and willing to think hard about consequences, when answering the preferences assessment questions, and if necessary, reconsider to achieve a consistent set of preferences. This multi-attribute utility model is far more complicated and elaborate than the value model, and can be used as a second and more thorough approach to the stakeholder's preferences and risk attitude against uncertainties.

\section{UAS Design Integration}

Isight [1] was chosen as the integrating design tool of all models because of its ability to execute simulation-based processes in a visual and flexible way, allowing use and control of various software components utilized in the design process. Following the integration of all models, the design space is explored without setting any constraints using the techniques of Design of Experiments (DoE). For multivariable optimization trade studies, the workload increases exponentially as the number of the design variables goes up. To keep the number of them to a tractable number, several design variables were selected to be varied along with their corresponding ranges, such as wing span $(1.25-1.75 \mathrm{~m})$, wing aspect ratio (6-12), battery capacity (6-10 Ahr) etc. The rest of them were set at reasonable values: wing twist $2^{o}$, wing sweep of $15^{\circ}$ for the flying wing, and so on. However, they too could vary if desired, for further design space exploration. For the DoE, three levels were chosen for the design variables, i.e. for wing aspect ratio 6, 9, 12 and for battery capacity 6.5, 8, 9.5. The DoE's performed were Full Factorial allowing for all possible interactions to be evaluated, as well as Latin Hypercube for more random combinations' generation, with varying number of experiments. Based on the results obtained from the DoE, the UAS was optimized for value and utility, depending on which model was used. Moreover, sensitivity analysis and trade-off studies between design parameters and results were performed. For the optimization, Isight allows several techniques to be implemented. The Hooke-Jeeves Direct Search was selected, since it is suitable for non-linear design spaces and long running simulations.

The design space exploration aimed at maximizing value or utility index or alternatively optimizing some critical attribute, such as operational surveillance time, maximum endurance/maximum range achieved, or total lifecycle cost. 
The preferences/priorities of the user, as reflected in the value/utility models, are critical for the identification of the optimal design and can indeed provide different results. Thus, based on a user with 'balanced' priorities between performance (endurance, range) and lifecycle cost (acquisition and through-life) and a 'military' user, focusing mostly on maximizing survivability, minimizing UAS detectability and maximizing data collection capabilities, different aircraft optimal designs were obtained. For a user with 'balanced' priorities an aircraft with V-shaped tail and wing span of $1.5 \mathrm{~m}$ was identified as optimal, whilst for a 'military' user the UAV with T-shaped tail and a wing span of $1.25 \mathrm{~m}$ was the optimal configuration.

Based on the user's specific preferences, dominant aircraft configurations/geometries maximizing value or utility index are identified, as in Fig. 6. The monocoque fuselage, V-shape tail pusher propeller configuration, followed by the monocoque fuselage, Y-shape tail, pusher propeller configuration and monocoque fuselage, T-shape tail, pusher propeller configuration are dominating in terms of both value and utility indices.

Additionally, the optimum range of design variables was obtained, while surface plots demonstrate the effect of design variables or other parameters on the response, as in Fig. 7. Both value and utility models point to selecting the same ranges of design variables, such as a wing aspect ratio of around 12 , a wing span of $1.5 \mathrm{~m}$, a battery capacity of 9.5Ahr, and an intermediate scheduled maintenance interval, while for some design variables with small influence, such as fin aspect ratio and horizontal tail aspect ratio, their optimal ranges are not clear.

Alternatively, optimizing for some critical aircraft attribute, the twin boom, inverted V-shape tail and tractor propeller configuration was identified as the dominant both for operational surveillance time when flying at design speed and total lifecycle cost. For maximum endurance, the flying wing with pusher propeller aircraft was found as expected to be the dominant one with wing aspect ratio of 12 and wing span of $1.25 \mathrm{~m}$, while for maximum range, a flying wing, pusher propeller configuration with wing aspect ratio of 6 and wing span of $1.25 \mathrm{~m}$ is the optimum one.

Hence, the multi-attribute value model provides analogous results with the multi-attribute utility model and can address effectively the user's preferences. Both models identify the same aircraft configurations as dominant in terms of maximizing value or utility index, while in the surface plots, they capture similar effects of design variables on the response, value or utility. 


\section{Discussion and Conclusions}

The main objective of this research has been to develop a framework that will apply the value driven design philosophy in the conceptual design phase of a defence UAS through a multi-criteria decision making analysis. The following aspects have been considered:

- The conceptual VDD framework generates systematically, following a novel geometric topologies parameterization instead of dealing with a single concept, defines, analyses the aircraft design points, and calculates their value, based on the assessment of a single stakeholder/user's needs or preferences.

- The VDD framework, performing an extensive design exploration and multidisciplinary design optimisation, achieves automated design identification, relaxing all requirements and maximizing the value/utility objective function as advocated by the VDD.

- The novel multi-attribute norm value model is successfully employed as a basic value model during the conceptual phase design, capturing the stakeholder's needs, defining objectively the set of optimal design alternatives, framing and guiding engineering design. The problem of using an unjustifiable numerical scale in AHP for the calculation of weighting factors is also tackled through this value model.

- The multi-attribute utility model has manifested the extensive interaction with the user, required to establish and assess their preferences concerning the consequences. This model could be used as a second and more thorough approach to the stakeholder's preferences and risk attitude against uncertainties, once the list of the design alternatives is finalized in the latter stages of the design.

Current and future work includes the application/testing of this framework in a practical case, capturing customer preferences/needs and trade-offs between them, and assessing alternative aircraft configurations and technologies.

\section{Acknowledgments}

This work has been conducted through the Southampton University Engineering Centre of Excellence. The authors would like to thank the Defence Science and Technology Laboratory (DSTL) for the funding and support of this project. 


\section{References}

1. Isight \& the Simulia Execution Engine. Available from: http://www.3ds.com/productsservices/simulia/portfolio/isight-simulia-execution-engine/overview/.

2. www.merriam-webster.com/dictionary.

3. $\quad$ Systems Engineering Handbook Version 3. 2006: International Council on Systems Engineering.

4. $\quad$ Antonsson, E.K., Cagan, J., Formal Engineering Design Synthesis. 2001: Cambridge University Press.

5. Butler, J., D.J. Morrice, and P.W. Mullarkey, A Multiple Attribute Utility Theory Approach to Ranking and Selection. Management Science, 2001. 47(6): p. 800-816.

6. Castagne, S., R. Curran, and P. Collopy, Implementation of value-driven optimisation for the design of aircraft fuselage panels. International Journal of Production Economics, 2009. 117(2): p. 381-388.

7. Chang, Y.H. and C.H. Yeh, Evaluating airline competitiveness using multi-attribute decision making. Omega, 2001. 29: p. 405-415.

8. Chen, Y.M., Huang,P-N, Bi-negotiation Integrated AHP in Suppliers Selection. International Journal of Operations \& Production Management, 2007. 27(11): p. 1254-1274.

9. Cheung, J., et al., Application of Value-Driven Design to Commercial Aeroengine Systems. Journal of Aircraft, 2012. 49(3): p. 688-702.

10. Collopy, P.D., Aerospace System Value Models: A Survey and Observations, in AIAA Space 2009 Conference \& Exposition. 2009, AIAA: Pasadena, Ca, USA.

11. Collopy, P.D. and P.M. Hollingsworth, Value-Driven Design. Journal of Aircraft, 2011. 48(3): p. 749-759.

12. $\quad$ Cross, N., Engineering Design Methods: Strategies for Product Design. 3rd ed. 2000: Chichester: Wiley.

13. Crossman, N.D., Bryan,B.A., King, D., Contribution of Site Assessment Toward Prioritising Investment in Natural Capital. Environmental Modelling \& Software, 2011. 26: p. 30-37.

14. Dyer, J.S., Remarks on the Analytic Hierarchy Process. Management Science, 1990. 36(3): p. 249-258.

15. Dyer, J.S. and R.K. Sarin, Measurable Multiattribute Value Functions. Operations Research, 1979. 27(4): p. 810-822.

16. Edwards, W., How to use multiattribute utility measurement for social decision making. A Science of Decision Making: The Legacy of Ward Edwards, ed. J.W. Weiss, Weis,D.J. 2009, New York, USA: Oxford University Press. 511.

17. Edwards, W. and F.H. Barron, SMARTS and SMARTER: Improved Simple Methods for Multiattribute Utility Measurement. Organizational Behavior and Human Decision Processes, 1994. 60(3): p. 306-325.

18. Eggert, R.J., Engineering Design. 2004, Upper Saddle River, NJ: Pearson Prentice Hall, USA.

19. Elliot, M.A., Selecting Numerical Scales for Pairwise Comparisons. Reliability Engineering and System Safety, 2010. 95: p. 750-763.

20. Eres, M.H., et al., Mapping Customer Needs to Engineering Characteristics: An Aerospace Perspective for Conceptual Design. Journal of Engineering Design, 2014. 25(1-3): p. 64-87.

21. Fishburn, P.C., Utility Theory for Decision Making. 1970, New York: John Wiley \& Sons.

22. Hazelrigg, G.A., Systems Engineering: An Approach to Information-Based Design. 1996: Prentice-Hall, Upper Saddle River, NJ. p. pp. 158-161, 291-311.

23. Hazelrigg, G.A., Validation of Engineering Design Alternative Selection Methods. Journal of Engineering Optimization, 2003. 35(2): p. 103-120.

24. Hubka, V., Eder,W.E., A Total Concept Theory for Engineering Design. 1988, New York: NY:Springer.

25. Keeney, R.L., Value-focused Thinking: a Path to Creative Decision Making. 1996, Cambridge, MA Harvard.

26. Keeney, R.L. and H. Raiffa, Decisions with Multiple Objectives. 1976, Cambridge, UK: Press Syndicate of the Cambridge University.

27. Kirkwood, C.W. and J.L. Corner, The effectiveness of partial information about attribute weights for ranking alternatives in multiattribute decision making. Organizational Behavior and Human Decision Processes, 1993. 54(3): p. 456-476.

28. Lai, V.S., B.K. Wong, and W. Cheung, Group Decision Making in a multiple criteria environment: A case using the AHP in software selection. European Journal of Operational Research, 2002. 137: p. 134-144.

29. Otto, K.N., Wood,K.L., Product Design:Techniques in Reverse Engineering and New Product Development. 2001, Upper Saddle River,NJ: Prentice Hall.

30. Pahl, G., Beitz, W., Engineering Design: A Systematic Approach. 3rd ed. 2007, London: Springer-Verlag. 617.

31. Perez, J., Some Comments on Saaty's AHP. Management Science, 1995. 41(5): p. 1091-1095. 
32. Pugh, S., Total Design: Integrated Methods for Successful Product Engineering. 1991, Wokingham, England: Addison-Wesley Publishing Company.

33. Raymer, D.P., Multivariable Aircraft Optimisation on a Personal Computer, in SAE/AIAA. 1996, AIAA.

34. Raymer, D.P., Aircraft Design: A conceptual Approach. 5th ed. 2012, Virginia, USA: AIAA Education Series.

35. Ross, A.M., et al., Aligning Perspectives and Methods for Value-Driven Design, in AIAA Space, AIAA, Editor. 2010, AIAA: Anaheim, Ca, USA.

36. Roy, B., The Outranking Approach and the Foundations of ELECTRE methods. Theory and Decision, 1991. 31(1): p. 49-73.

37. Saaty, T.L., The Analytic Hierarch Process. 1980, New York, USA: McGraw-Hill.

38. Saaty, T.L. and L.G. Vargas, Models, Methods, Concepts and Applications of the Analytic Hierarchy Process. 2001, Masschusetts, USA: Kluwer Academic Publishers.

39. Scholl, A., Manthey, L., Helm,R., Steiner, M., Solving Multi-attribute Design Problems with AHP and Cojoint Analusis: An Empirical Comparison. European Journal of Operational Research, 2005. 164: p. 760777.

40. Sha, D.Y., Che,Z.H., Virtual Integration with a Multi-Criteria Partner Selection Model for the Multi-Echelon Manufacturing System. International Journal of Advanced Manufacturing Technology, 2005. 25: p. 793-802.

41. Sobester, A., Four Suggestions for Better Parametric Geometries, in 10th AIAA Multidisciplinary Design Optimization Conference. 2014. p. 10.

42. Tarek, Z. and M. Elsayed, Budget Allocation and Rehabilitation Plans for Water Systems Using Simulation Approach. Tunnelling and Underground Space Technology, 2013. 36: p. 34-45.

43. Ullman, D.G., The Mechanical Design Process. 2009, New York: NY:McGraw-Hill Higher Education.

44. Ulrich, K., Eppinger,S., Product Design and Development. 4th ed. 2008, New York: NY: McGraw-Hill.

45. Von Neumann, J. and O. Morgenstern, Theory of Games and Economic Behavior. 3rd ed. 1953, Princeton,NJ: Princeton University Press.

46. Wiese, P.R., Engineering Design in the Multi-Discipline Era, A system approach. 2003, UK: Professional Engineering Publishing Limited.

47. Yoon, K.P. and C.L. Hwang, Multiple Attribute Decision Making. An Introduction. Quantitative Applications in the Social Sciences. Vol. 104. 1995, USA: Sage Publications Inc. 76. 
Table 1 Value Functions

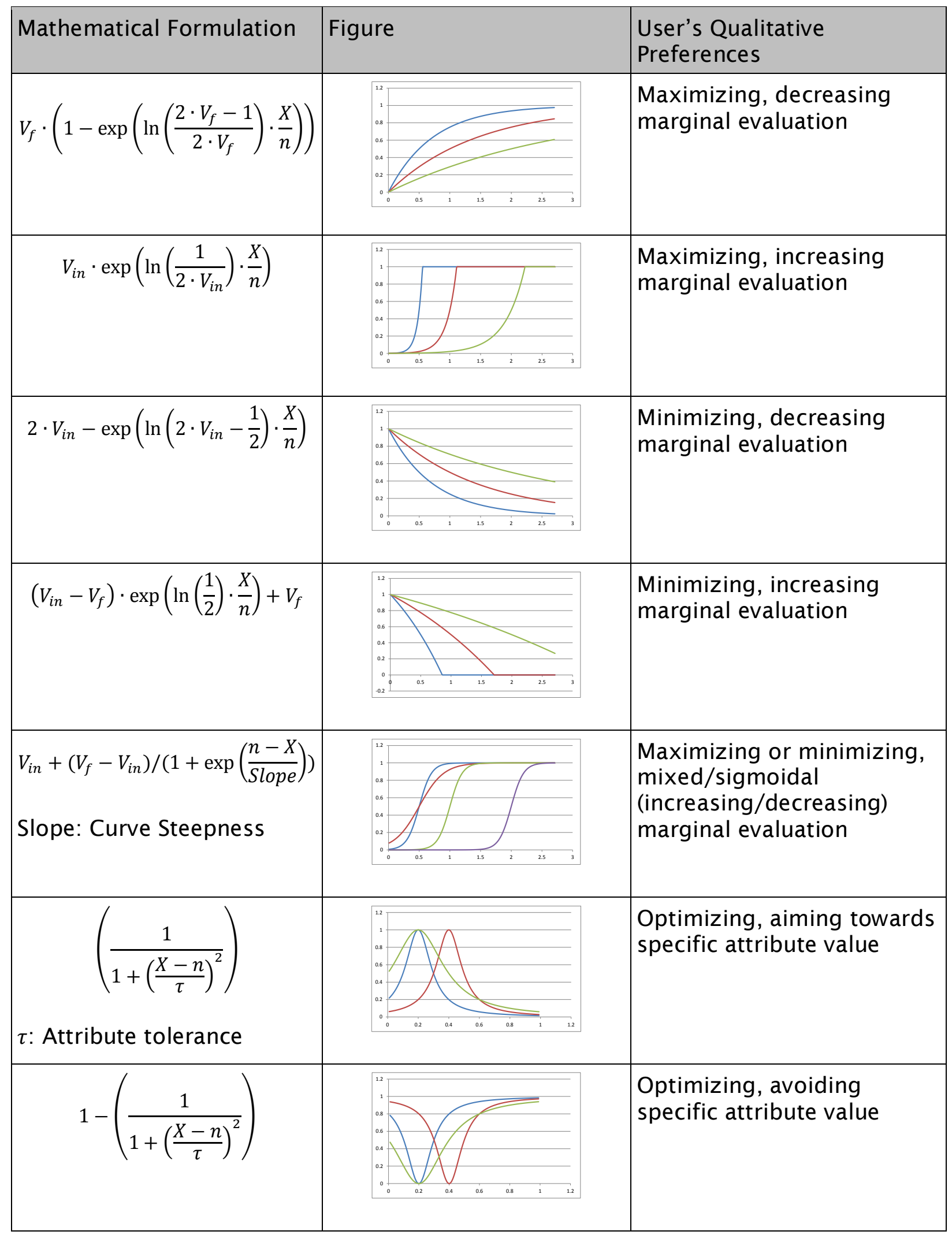


Table 2 AHP Numerical Scales

\begin{tabular}{|l|c|c|l|}
\hline \multicolumn{1}{|c|}{ Definition } & $\begin{array}{c}\text { Integer } \\
\text { Scale }\end{array}$ & $\begin{array}{c}\text { Power } \\
\text { Scale }\end{array}$ & \multicolumn{1}{c|}{ Explanation } \\
\hline Equal importance & 1 & 1 & Two factors contribute equally \\
\hline $\begin{array}{l}\text { Somewhat more } \\
\text { important }\end{array}$ & 3 & 1.73 & Slightly favour one over the other \\
\hline Much more important & 5 & 3 & Strongly favour one over the other \\
\hline $\begin{array}{l}\text { Very much more } \\
\text { important }\end{array}$ & 7 & 5.2 & Very strongly favour one over the other \\
\hline $\begin{array}{l}\text { Absolute more } \\
\text { important }\end{array}$ & 9 & 9 & $\begin{array}{l}\text { Highest possible validity of favouring one over the } \\
\text { other }\end{array}$ \\
\hline
\end{tabular}


Table 3 AHP Weighting Factors Assessment

\begin{tabular}{|c|c|c|c|c|c|c|c|c|c|c|c|c|c|c|}
\hline User Needs & 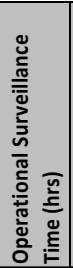 & 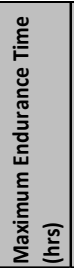 & 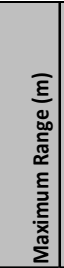 & 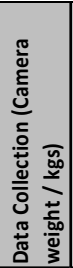 & 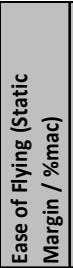 & 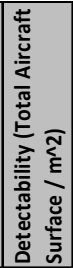 & 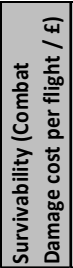 & 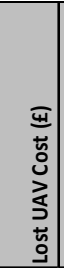 & 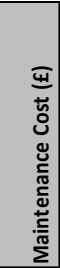 & 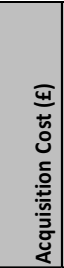 & 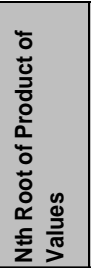 & 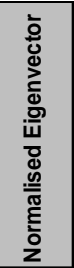 & 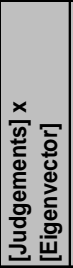 & 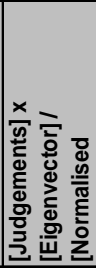 \\
\hline Operational Surveillance Time (hrs) & 1.000 & 1.333 & 1.333 & 2.000 & 2.000 & 4.000 & 4.000 & 2.000 & 2.000 & 1.333 & 1.898041 & $17.64 \%$ & 1.7767 & 10.06992 \\
\hline Maximum Endurance Time (hrs) & 0.750 & 1.000 & 1.000 & 1.333 & 1.333 & 2.000 & 4.000 & 1.333 & 1.333 & 1.333 & 1.381289 & $12.84 \%$ & 1.292 & 10.06194 \\
\hline Maximum Range (m) & 0.750 & 1.000 & 1.000 & 1.333 & 1.333 & 2.000 & 4.000 & 1.333 & 1.333 & 1.333 & 1.381289 & $12.84 \%$ & 1.292 & 10.06194 \\
\hline Data Collection (Camera weight / kgs) & 0.500 & 0.750 & 0.750 & 1.000 & 1.000 & 1.333 & 2.000 & 1.333 & 1.333 & 0.800 & 1.006475 & $9.36 \%$ & 0.9416 & 10.06436 \\
\hline Ease of Flying (Static Margin / \%mac) & 0.500 & 0.750 & 0.750 & 1.000 & 1.000 & 1.333 & 2.000 & 1.333 & 1.333 & 0.800 & 1.006475 & $9.36 \%$ & 0.9416 & 10.06436 \\
\hline Detectability (Total Aircraft Surface $/ \mathbf{m}^{\wedge} \mathbf{2}$ ) & 0.250 & 0.500 & 0.500 & 0.750 & 0.750 & 1.000 & 1.333 & 0.667 & 0.667 & 0.500 & 0.633538 & $5.89 \%$ & 0.594 & 10.08553 \\
\hline Survivability (Combat Damage cost per flight / $£$ ) & 0.250 & 0.250 & 0.250 & 0.500 & 0.500 & 0.750 & 1.000 & 0.500 & 0.500 & 0.333 & 0.435275 & $4.05 \%$ & 0.4081 & 10.08484 \\
\hline Lost UAV Cost (f) & 0.500 & 0.750 & 0.750 & 0.750 & 0.750 & 1.500 & 2.000 & 1.000 & 1.000 & 0.800 & 0.907701 & $8.44 \%$ & 0.8484 & 10.05479 \\
\hline Maintenance Cost (f) & 0.500 & 0.750 & 0.750 & 0.750 & 0.750 & 1.500 & 2.000 & 1.000 & 1.000 & 0.800 & 0.907701 & $8.44 \%$ & 0.8484 & 10.05479 \\
\hline Acquisition Cost (f) & 0.750 & 0.750 & 0.750 & 1.250 & 1.250 & 2.000 & 3.000 & 1.250 & 1.250 & 1.000 & 1.199769 & $11.15 \%$ & 1.1205 & 10.04658 \\
\hline 10 & & & & & & & & & & & 10.75755 & 1 & MEAN & 10.08553 \\
\hline & & & & & & & & & & & & 0.009503 \\
\hline & & & & & & & & & & & \multicolumn{3}{|c|}{$\begin{array}{r}\text { CONSISTENCYINDEX } \\
\text { RANDOMCI }\end{array}$} & 0.553 \\
\hline & & & & & & & & & & & \multicolumn{3}{|c|}{ CONSISTENCY RATIO } & $1.72 \%$ \\
\hline
\end{tabular}


Fig. 1 Engineering Design Process [23]

Fig. 2 UAS Objectives/Attributes Hierarchy

Fig. 3 UAS Value Driven Design Cycle

Fig. 4 Aircraft Geometric Topologies Generation

Fig. 5 Weights Distribution of AHP Scales

Fig. 6 Comparison of Aircraft Configurations

Fig. 7 Surface Plot of Value Index as a Function of Wing Span and Wing Aspect Ratio 


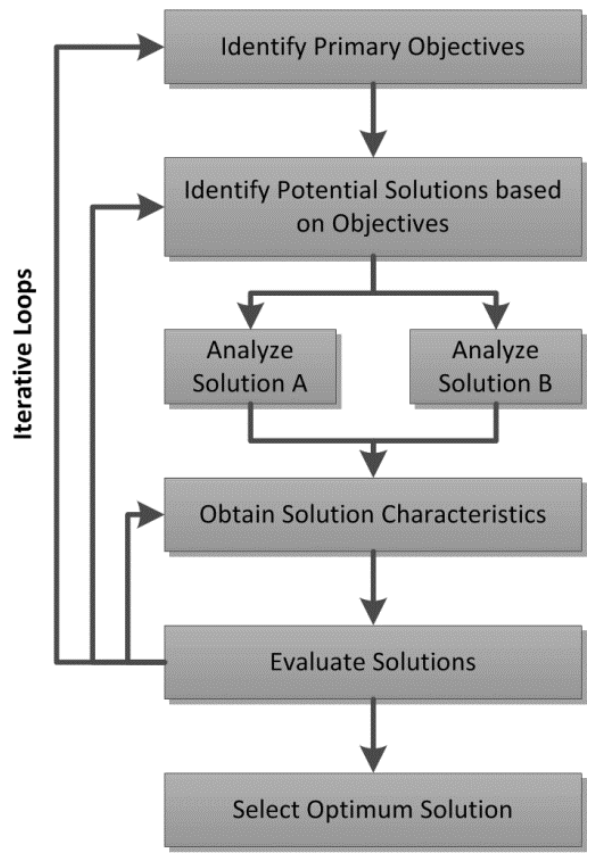

Fig. 1 Engineering Design Process [23] 


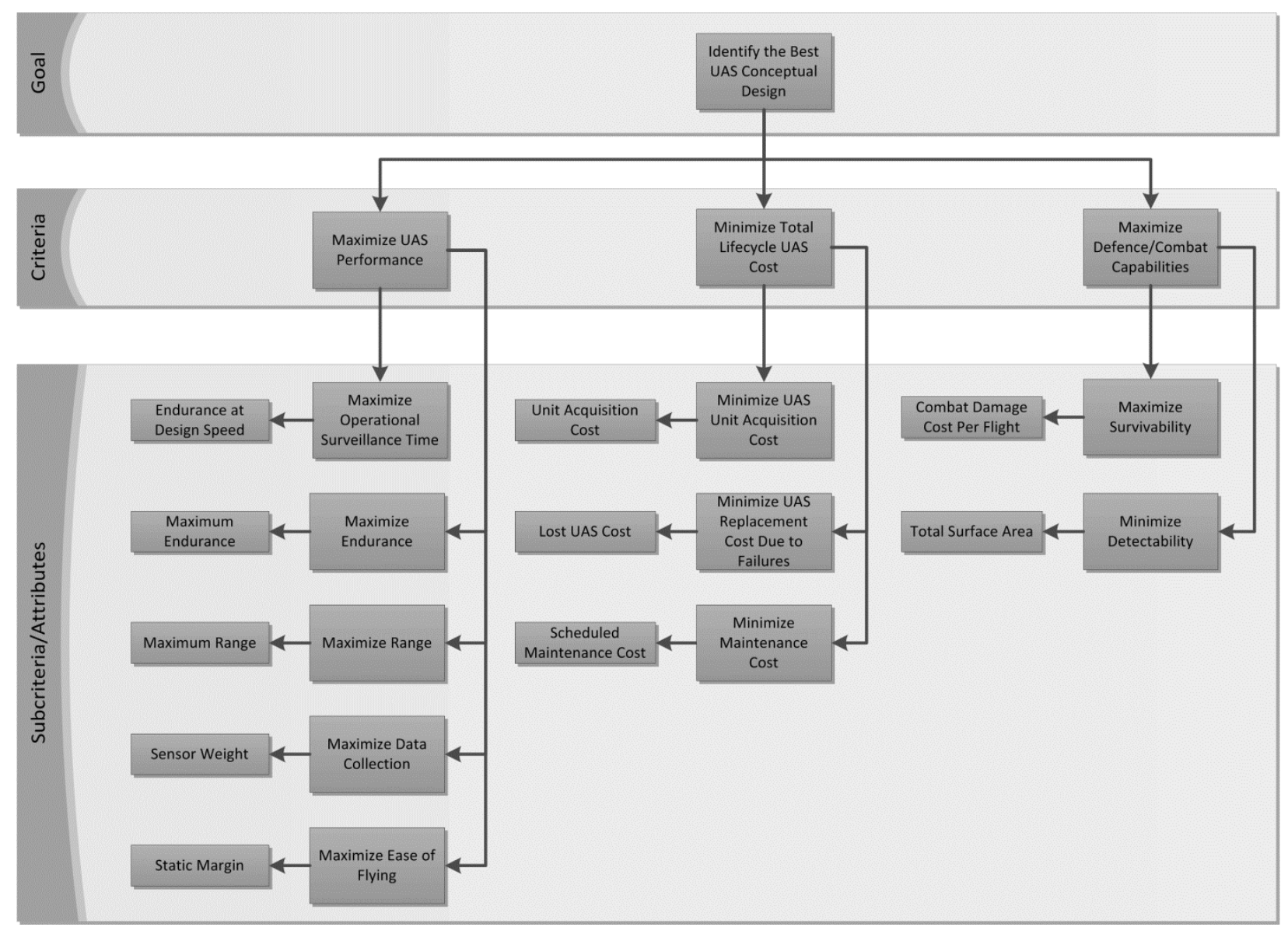

Fig. 2 UAS Objectives/Attributes Hierarchy 


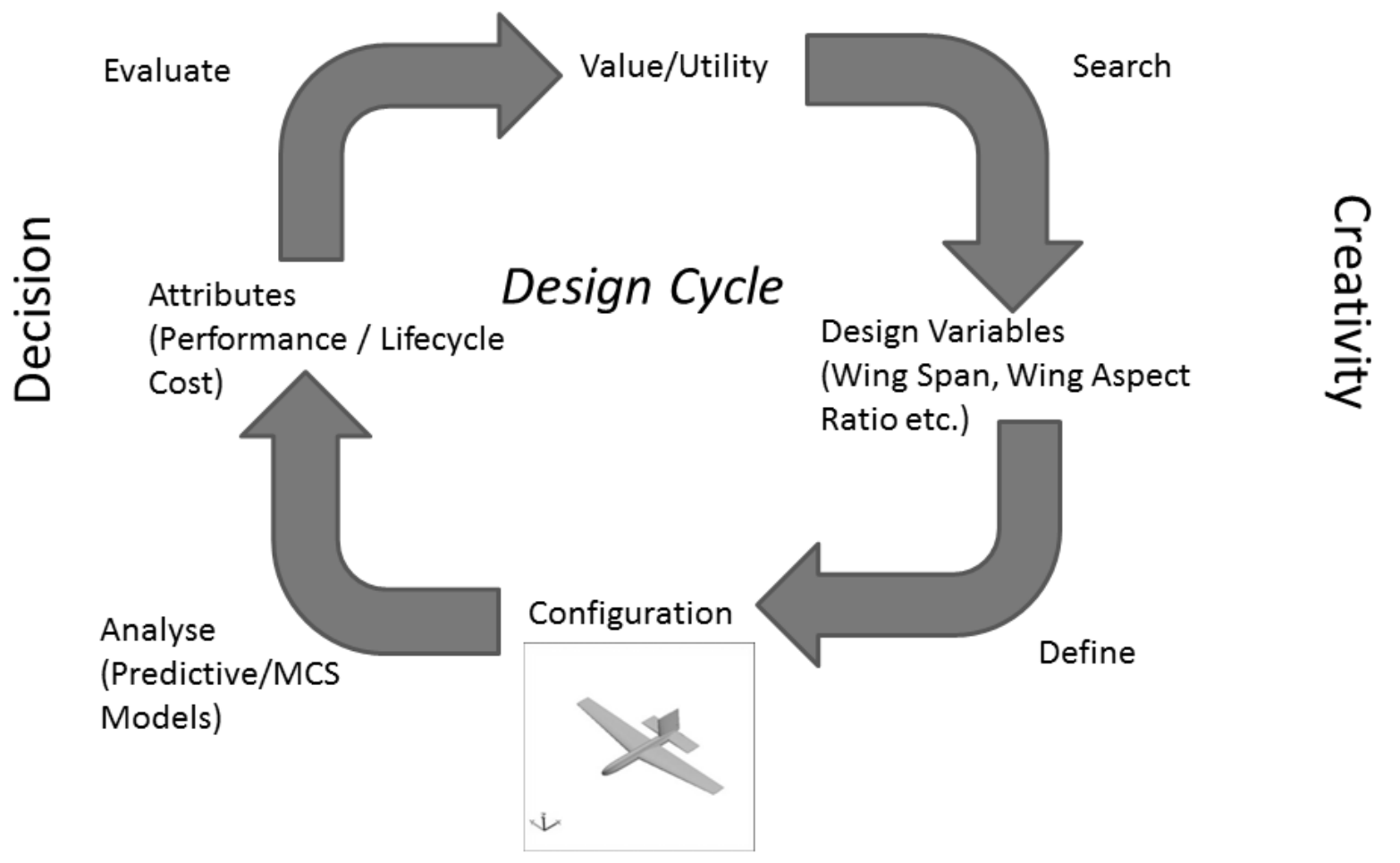

Fig. 3 UAS Value Driven Design Cycle 


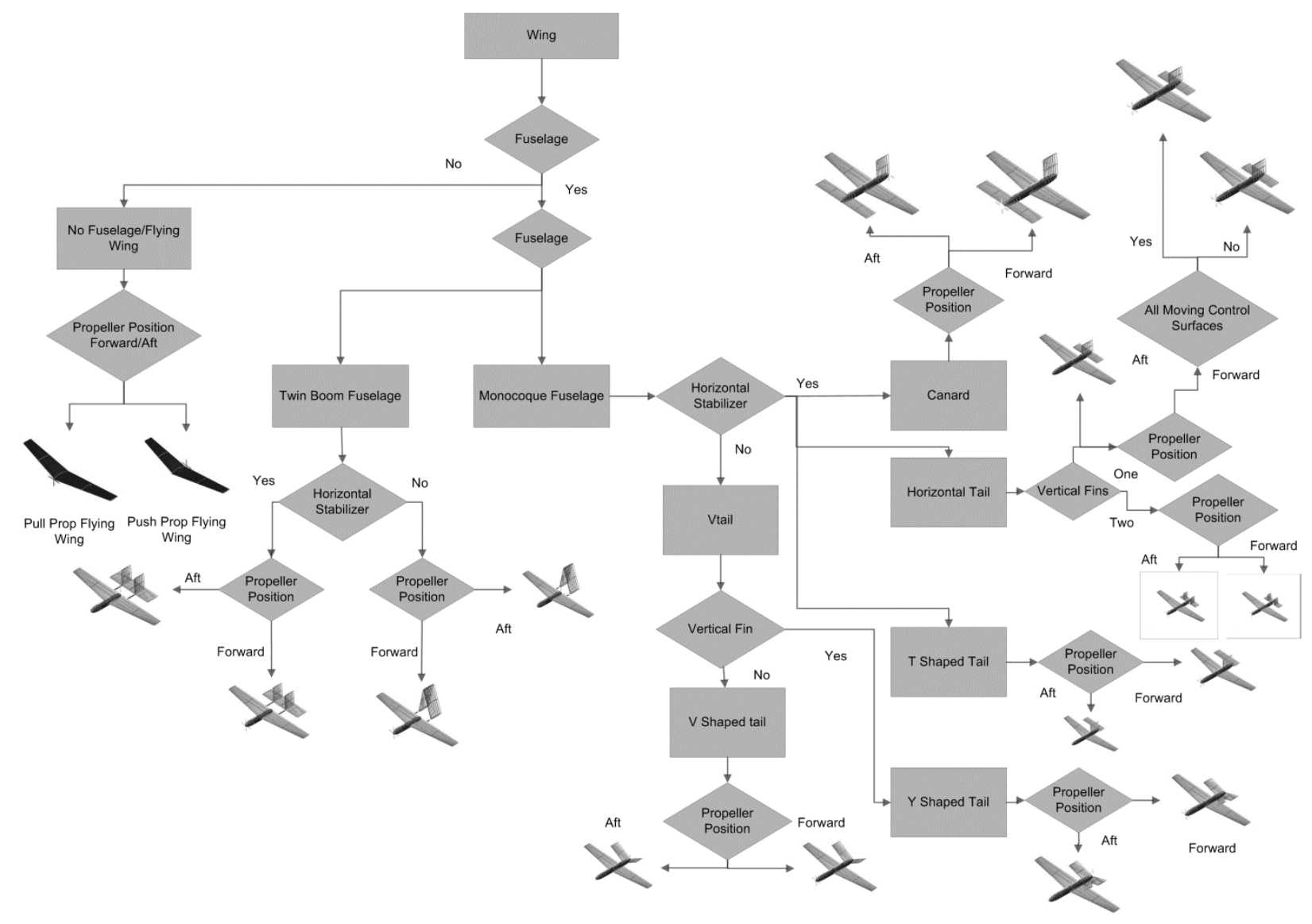

Fig. 4 Aircraft Geometric Topologies Generation 


$$
\triangle D
$$




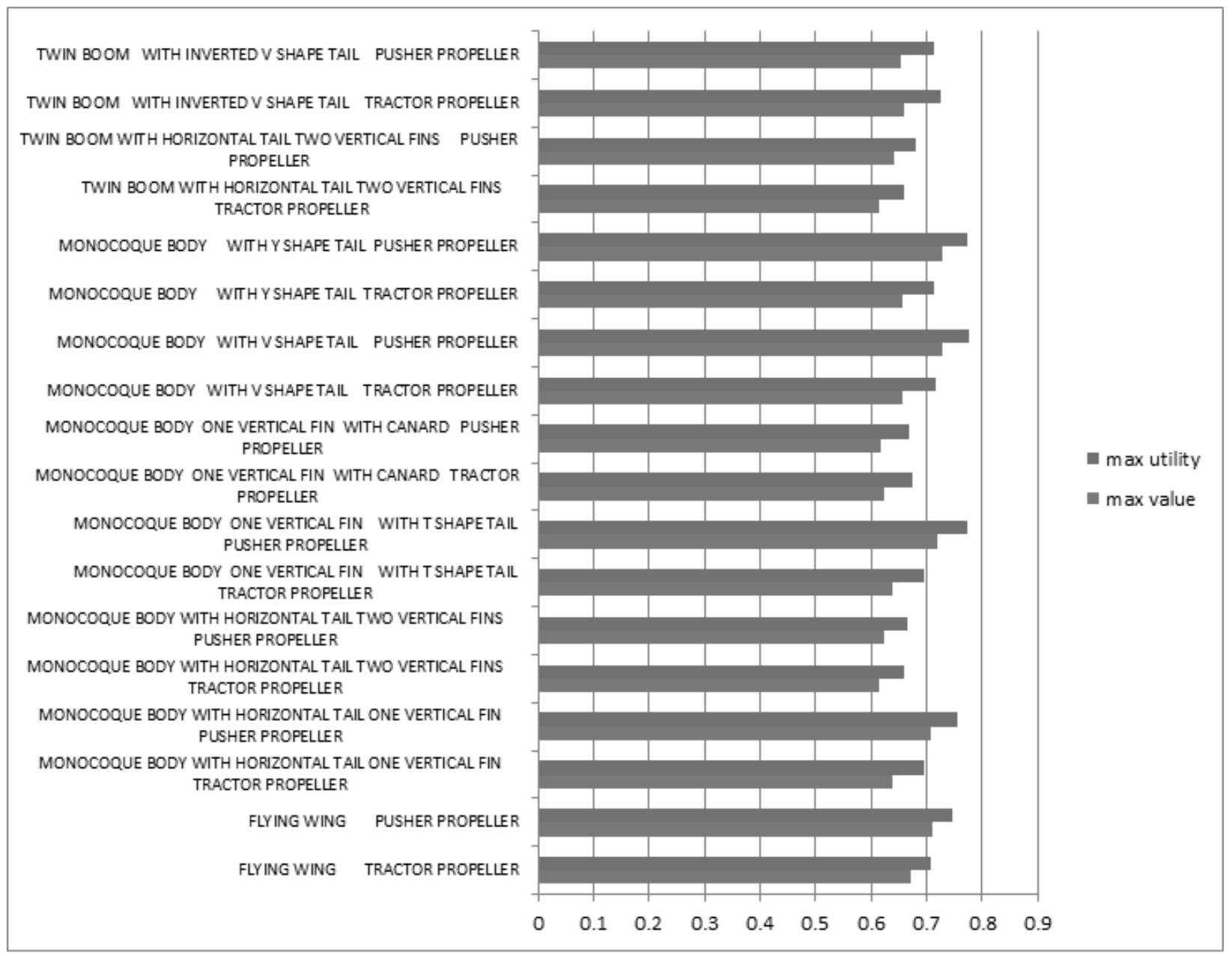

Fig. 6 Comparison of Aircraft Configurations 


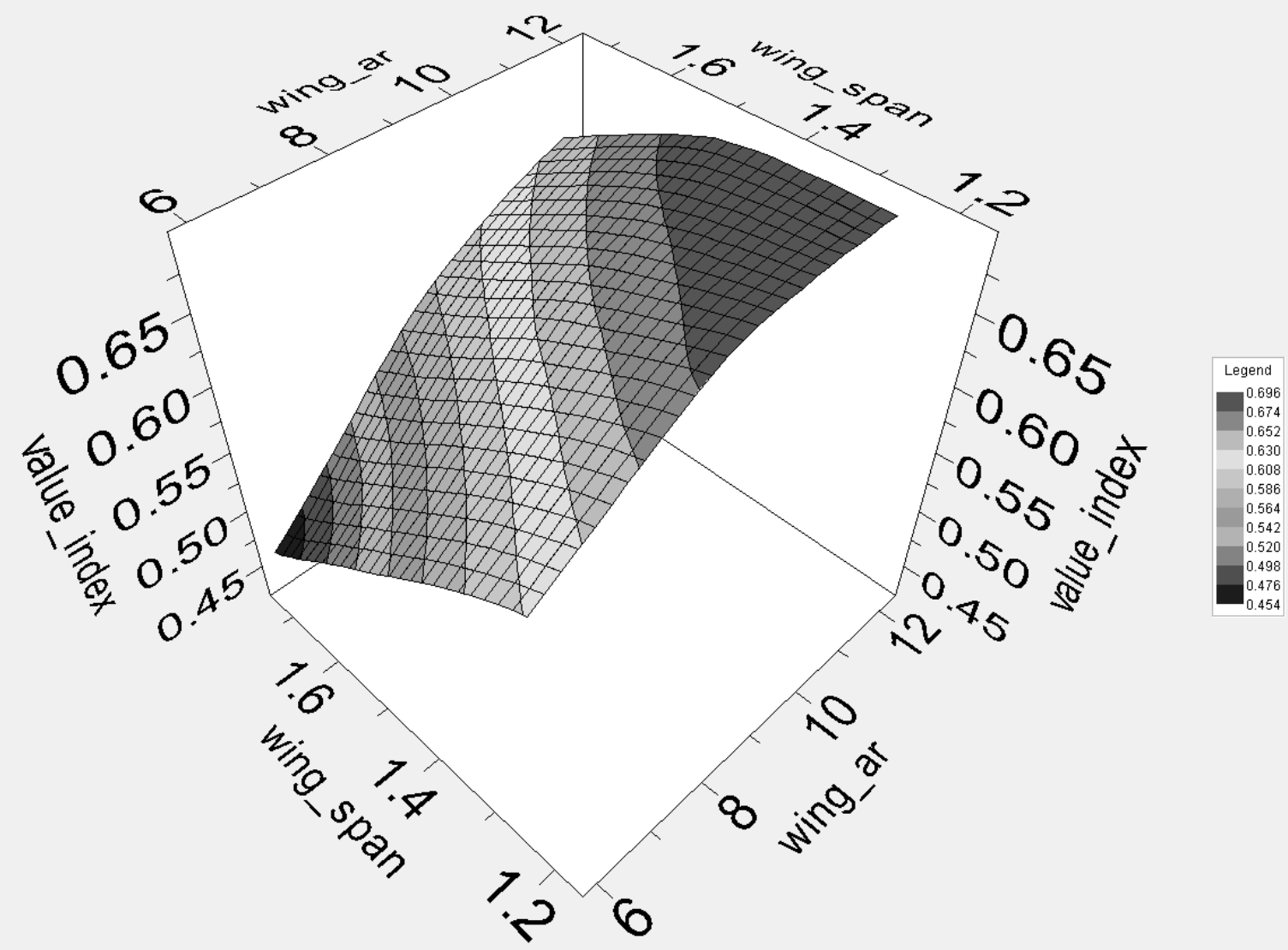

Fig. 7 Surface Plot of Value Index as a Function of Wing Span and Wing Aspect Ratio 